\title{
Propuestas didácticas mediadas por tecnologías digitales para el desarrollo de competencias de lectura y escritura académicas
}

\author{
Guadalupe Álvarez' \\ Consejo Nacional de Investigaciones Científicas y Técnicas, CONICET \\ Universidad Nacional de General Sarmiento (Argentina) \\ María Beatriz Taboada² \\ Universidad Autónoma de Entre Ríos (Argentina) \\ Consejo Nacional de Investigaciones Científicas y Técnicas, CONICET
}

Recibido: julio20 de 2016. Revisado: septiembre 5 de 2016. Aceptado: octubre 30 de 2016

Referencia formato APA: Álvarez, G., \& Taboada, M. B. (2016). Propuestas didácticas mediadas por tecnologías digitales para el desarrollo de competencias de lectura y escritura académicas. Rev.Guillermo de Ockham, 14(2), 83-91. doi: http://dx.doi.org/10.21500/22563202.2336

\section{Resumen}

En el marco de una investigación que buscar caracterizar y comprender prácticas de enseńanza de la escritura con tecnologías digitales que han tenido lugar en el nivel medio y superior en Argentina, el presente artículo describe y analiza dos experiencias educativa (en Facebook y Moodle, respectivamente) que han buscado contribuir al desarrollo de competencias de lectura y escritura para el nivel superior, a partir de diferentes herramientas y dispositivos en los que se integran nuevas tecnologías. El análisis de los casos se realiza teniendo en cuenta cuatro aspectos básicos: 1. los criterios con los cuales fueron planificadas y diseñadas las experiencias; 2. las diferentes modalidades de implementación; 3. las características que adoptaron las discusiones o la resolución de ejercicios de reformulación en el muro y en los ejercicios de Moodle, respectivamente; 4. el punto de vista de los estudiantes sobre dichos espacios. Aun cuando las experiencias son preliminares, su análisis brinda herramientas para repensar estrategias didácticas para integrar tecnologías digitales en propuestas de enseñanza-aprendizaje que apuntan a resolver problemáticas propias de la alfabetización académica.

Palabras clave: Universidad, alfabetización académica, tecnologías digitales

\section{Didactic proposals mediated by digital technologies for the development of academic reading and writing skills}

\section{Abstract}

As part of an investigation which is aimed at characterizing and understanding practices of teaching writing with digital technologies in the middle and upper level in Argentina, this paper describes and analyzes two educational experiences

1. Doctora en Letras. Profesora y Licenciada en Letras. Dirige actualmente el proyecto "Las prácticas de enseńanza de la escritura mediadas por tecnologías digitales. Estudio de casos en los niveles medio y superior en Argentina”, en la Universidad Nacional de General Sarmiento. Dirección postal: Monroe 1181 (1428) Ciudad de Buenos Aires, Argentina. Correo electrónico: galvarez@ungs.edu.ar

2. Doctora en Humanidades y Artes, mención Lingüística. Magíster en Enseñanza de la Lengua y la Literatura. Magíster en Filología Hispánica. Profesora de Enseńanza Superior en Castellano, Literatura y Latín. Profesora en Letras. Directora del proyecto "Alfabetización avanza y académica: espacios para la reflexión y la formación". Dirección postal: Jordana 373, (3260) Concepción del Uruguay, Entre Ríos, Argentina. Correo electrónico: mbtaboada@conicet.gov.ar 
(on Facebook and Moodle, respectively) which have been designed to develop academic reading and writing skills by using different tools and devices that integrate new technologies. The case analysis is performed taking into account four basic dimensions: 1) the criteria by which the experiences were planned and designed; 2) different modalities of implementation; 3) the characteristics adopted in reformulation exercises on the wall and Moodle, respectively; 4) the point of view of students on such spaces. Even when experiences are preliminary analysis, they provides tools to rethink teaching strategies in order to integrate digital technologies in teaching and learning proposals aimed at improving academic literacy.

Keywords: University, academic literacy, digital technologies

\section{Propostas didáticas mediadas pôr tecnologias digitales para o desenvolvimento competências de leitura e escrita acadêmica}

\section{Resumo}

No marco de uma pesquisa que visa caracterizar e compreender práticas de ensino da escrita com tecnologías digitais que tiveram lugar no nível meio e superior em Argentina, o presente artigo descreve y analise dois experiências educacionais (em Facebook e Moodle, respectivamente) que têm procurado ajudar a desevolver habilidades de leitura e escrita para o nível superior, a partir de diferentes ferramentas e dispositivos que integram novas tecnologias. $\mathrm{O}$ analise dos casos se realiza tendo em conta quatro aspectos básicos: 1 . Os criterios com os cuais foram planejadas e deseñadas as experiências. $s$ diferentes modalidades de execuçáo; 3. adoptou as discussóes características ou resolução de exercícios reformulaçáo na parede e exerce Moodle, respectivamente; 4. Do ponto de vista dos alunos sobre esses espaços. Ainda cuando as experiências são preliminares, seu analise fornece ferramentas para repensar estratégias didáticas para integrar tecnologias digitais em propostas de ensino-aprendizagem no sentido de resolver os seus próprios problemas de estratégias de alfabetização acadêmicos.

Palavras-chave: Universidade, alfabetização acadêmica, tecnologias digitais

\section{Introducción}

En los últimos años se han multiplicado las investigaciones que ponen en evidencia las diversas dificultades que los estudiantes enfrentan para resolver las tareas de lectura y escritura que la universidad les propone, tanto en sus instancias de ingreso (Narvaja de Arnoux et al., 2002; García \& Álvarez, 2009, 2010), como en el desarrollo de las carreras (Estienne \& Carlino, 2004; Lacon de De Lucia \& Ortega de Hocevar, 2004; Vázquez \& Miras, 2004; Carlino 2005; Piacente \& Tittarelli, 2006).

Frente a lo expuesto y aun cuando numerosas universidades argentinas todavía enfrentan estas situaciones por medio de esfuerzos aislados, es posible relevar propuestas que abordan de modo integral los problemas mencionados (Martínez, 2002; Lacon de De Lucia \& Ortega de Hocevar, 2004; Parodi, 2003, 2005; Carlino 2005; García \& Álvarez, 2010; Taboada et al., 2010; Taboada, 2011). En este contexto, se han comenzado a indagar las potencialidades de las tecnologías de la información y la comunicación (TIC) en relación con el desarrollo de las competencias en lectura y escritura (Martínez, 2006; Reale, 2008; Álvarez, García \& Qués, 2010).

Sin embargo, varias investigaciones (Álvarez \& Álvarez Cadavid, 2012; Laurillard, 2010; Rey Valzacchi, 2010; Sosa, 2009; Torres \& Rama, 2010) revelan que, aún cuando la formación se haga de manera completamente virtual o integre tecnologías digitales a las prácticas de enseñanza presencial, el diseño de gran parte de los entornos y los materiales didácticos digitales parecería basado en una lógica textual, determinada por la cultura de la página impresa, sin que se registre un aprovechamiento adecuado de todas las potencialidades que brindan los medios electrónicos, como la multimedialidad, la hipertextualidad o la interactividad. Por otra parte, en muchos casos tampoco se observa una integración planificada de las herramientas o los materiales, en cuanto son escasos o casi nulos el diseño de las situaciones de enseñanza y aprendizaje, la distribución adecuada de los contenidos y 
las orientaciones hacia las necesidades de los estudiantes (Rodríguez Illera \& Escofet, 2006).

Es indudable que, ante los nuevos contextos educativos que emergen con la profundización de las plataformas virtuales (Gros, Salvat, 2000, 2004; Harasim et al., 1998) y, más recientemente, con la irrupción y rápida consolidación de la Web 2.0 o Web social (Bonaiuti, 2006; Granieri, 2005), se requiere de la implementación de estrategias docentes novedosas, capaces de suscitar, entre otros aspectos, la reflexión, la cooperación y el intercambio entre los alumnos. Estas estrategias deberían contemplar e integrar las diferentes potencialidades discursivas y comunicativas de los medios electrónicos (Avgerinou, 2009; Baldry \& Thibault, 2006; Gros Salvat, 2000, 2004; Kress, 2004, 2010; Salaverría, 2001; Scolari, 2004, 2008, 2009), como la multimedialidad, la hipertextualidad y el alto grado de interactividad propiciado en ambientes virtuales.

En función de estos supuestos y en el marco de una investigación ${ }^{3}$ que buscar caracterizar y comprender prácticas de enseñanza de la escritura con tecnologías digitales que han tenido lugar en el nivel medio y superior en Argentina, el presente artículo describe y analiza dos experiencias educativas que han buscado contribuir al desarrollo de competencias de lectura y escritura para el nivel superior, a partir de diferentes herramientas y dispositivos en los que se integran nuevas tecnologías.

\section{Metodología}

El enfoque metodológico de esta propuesta es netamente cualitativo y se enmarca en la tradición de estudios de casos múltiples (Miles y Huberman, 1991; Neiman y Quaranta, 2006; Vasilachis de Gialdino, 2006). Siguiendo esta tradición, buscamos llevar adelante un análisis que capture la complejidad de los casos específicos, permita hacer comparaciones entre los casos y llevar adelante contrastaciones que apoyen o desafíen las teorías vigentes acerca de la enseñanza de la escritura con tecnologías digitales en el nivel superior. Valiéndonos de este marco metodológico, seleccionamos dos experiencias de enseñanza de la escritura con tecnologías digitales en la universidad.

Los dos casos seleccionados corresponden a experiencias llevadas a cabo en el marco del taller de lectoescritura (TLE) de la Universidad Nacional de General Sarmiento (UNGS), asignatura que debe ser aprobada para ingresar a dicha universidad. Esta materia, totalmente presencial, hace especial hincapié en la comprensión y producción de textos explicativos y argumentativos.

En el marco esta asignatura, entonces, se desarrollaron dos experiencias que han tendido a superar problemas de los estudiantes preuniversitarios en las tareas de lectura y escritura académicas, incluidos aquellos relativos a la reformulación textual, mediante materiales especialmente diseñados para ser impartidos virtualmente. La primera de las experiencias relatadas consistió en un espacio en Facebook, en el cual se incluían ejercitaciones relativas a dicha práctica. La segunda tuvo lugar en Moodle e involucró materiales interactivos -que proponían prácticas de reformulación, pero no se reducían a estas- diseñados como estrategia de repaso para los parciales de la materia.

Para el relevamiento de los datos se hicieron entrevistas y encuestas a los participantes de las experiencias, incluidos los responsables y los estudiantes. También accedimos a los espacios virtuales en los cuales se llevaron a cabo las experiencias, así como a los materiales utilizados.

El estudio de los casos se efectuó basado en cuatro aspectos básicos: 1. los criterios con los cuales fueron planificadas y diseñadas las experiencias; 2. las diferentes modalidades de implementación; 3. las características que adoptaron las discusiones o la resolución de casos específicos de reformulación en el muro y en los ejercicios de Moodle, respectivamente, y 4 . el punto de vista de los estudiantes sobre dichos espacios. El análisis de estos aspectos nos ha permitido hacer una evaluación general de cada propuesta.

\section{Análisis de los casos}

\section{Espacio en Facebook del taller de lectoescritura}

\section{Diseńo de la propuesta}

La primera experiencia se hizo en Facebook (FB), a partir de un usuario llamado "taller de lectoescritura". La decisión de trabajar con $F B$ estuvo motivada, por un lado, por la gran cantidad de estudiantes que forman parte de esta red social y la utilizan diariamente para comunicarse con íntimos y conocidos. Además, desde el punto de vista didáctico, se consideró que las funcionalidades de esta red

3. Esta investigación, denominada Las prácticas de enseñanza de la escritura mediadas por tecnologías digitales. Estudio de casos en los niveles medio y superior en Argentina, se lleva a cabo con el aval de la Universidad Nacional de General Sarmiento, bajo la dirección de la doctora Guadalupe Álvarez y la codirección de la doctora Laura Ferrari. Los resultados del estudio permitirán delinear criterios para diseñar e implementar propuestas que mejoren la incorporación de tecnologías de la información y la comunicación (TIC) a la enseñanza de la escritura en diferentes niveles del sistema educativo. 
podrían contribuir con el tipo de actividades y discusiones propuestas (Llorens \& Capdeferro, 2011).

Una de las decisiones didácticas iniciales fue la conexión entre el espacio $F B$ y un blog en el cual se incluyeron materiales teóricos en diferentes formatos (fundamentalmente PDF) sobre los temas y contenidos involucrados en las actividades de reformulación. Por otra parte, se proyectó que el espacio en la red social sería utilizado también para poner a disposición materiales multimediales relevantes para la materia, proponer ejercicios y discusiones relativos a distintos casos de reformulación, compartir información general sobre la asignatura (por ejemplo, fecha de entrega de los trabajos prácticos), y enviar y recibir mensajes.

Sobre la base de las funciones mencionadas, el espacio $F B$ servía como soporte a las clases presenciales.

\section{Implementación de la propuesta}

Como el $F B$ se utilizaba por primera vez en la asignatura, solo participó una de las comisiones del TLE. La docente de esta comisión fue la responsable del espacio. Treinta y cinco de los cuarenta y dos alumnos se sumaron al espacio de la siguiente manera: el $50 \%$ de ellos, antes del primer parcial; el resto, con posterioridad.

Se registraron diferentes tipos de participaciones, así: mientras algunos estudiantes solo eran espectadores, otros intervenían activamente (mandaban mensajes, comentaban las publicaciones e, incluso, realizaban publicaciones).

En cuanto a los comentarios a las publicaciones de la docente, se verificó un intercambio interesante de los estudiantes en torno a diferentes casos de reformulaciones, un procedimiento fundamental de la escritura académica. En estos casos, si bien se presentaron solamente dos propuestas de análisis y la participación fue escasa, la dinámica, como veremos más adelante, resultó interesante.

Por otra parte, las participaciones de los estudiantes aumentaron ante la aparición de problemas "administrativos" (retiro de material en fotocopiadora, etc.) o inconvenientes con los trabajos prácticos o los contenidos de la materia.

Respecto de las publicaciones propuestas por los propios estudiantes, estas comprendieron materiales de internet sobre contenidos de la materia o consultas sobre cuestiones administrativas (horarios, materiales, etc.).

Hacia el final de la cursada, la participación de los estudiantes se incrementó e incluso intervinieron los que inicialmente no lo hacían. Por otra parte, los estudiantes dialogaban y discutían entre ellos sin la mediación del docente.

\section{Discusión de casos de reformulación a partir de publicaciones en el muro}

En el muro se planteó un ejercicio de reflexión y discusión sobre dos casos de reformulación. En la primera oportunidad, participaron dos estudiantes (a los que identificaremos como MJ y JV). En la segunda, solo uno (MJ). Como manifiesta esta observación, la participación en las discusiones no es una práctica frecuente entre los estudiantes. De hecho, suele intervenir el mismo grupo de estudiantes en todos los casos.

En cuanto a la dinámica del primer caso, la intervención de la docente decía lo siguiente:

"Para practicar para el parcial del lunes, les propongo leer la siguiente frase: 'La teoría de la evolución puede presentar malentendidos a poco de mencionar su nombre'. ¿Cómo reformularían la frase anterior si solo pudieran conservar en la reformulación las palabras 'la teoría de la evolución'?"

Este ejercicio, como se puede observar, apunta a que los estudiantes ejerciten diversas estrategias de reformulación (por ejemplo, cambio de orden y uso de sinónimos) y a que reconozcan el tipo de palabras o frases que no conviene modificar (en este caso, la denominación de una teoría científica).

Luego de esta intervención, participaron MJ y JV, sucesivamente y cada uno propuso su versión de la reformulación. Solo después de que la docente volvió a participar, los estudiantes intervinieron de acuerdo con lo dicho por su compañero.

En cuando a la dinámica del segundo caso, la intervención inicial de la docente decía:

"Seguimos practicando reformulación. ¿Cómo reformularían la siguiente frase?: 'La selección natural decide cuáles de las millones de variables que conforman un ser vivo son conservadas en el largo camino hacia la complejidad"”.

Tras esta intervención, se produce una seguidilla de participaciones en las cuales interactúan MJ, que propone versiones de la reformulación, y la docente, que revisa estas reformulaciones y señala errores o detalles mejorables. Finalmente, MJ escribe un mensaje personal (no público) a la docente en el que propone una versión de la reformulación y manifiesta sus dificultades para reformular. 


\section{Evaluación del curso por los estudiantes}

Al finalizar la cursada y mediante un mensaje privado de $F B$, se solicitó a los estudiantes que completaran un cuestionario a modo de encuesta. Las preguntas apuntaban a evaluar la frecuencia de visita a la plataforma, los materiales utilizados y las preferencias en relación con ellos. En este sentido, se preguntaba también sobre el uso del blog, los temas faltantes, la apreciación general sobre el sitio y los motivos por los cuales se había (o no) participado.

Un tercio de los estudiantes completaron este cuestionario y en relación con sus respuestas, dos aspectos llamaron nuestra atención. Por un lado, los estudiantes informaron que habían utilizado la red frecuentemente, incluso todos los días. En general, consultaban las publicaciones sobre materiales y ejercicios de reformulación y también aquellas relativas a la organización de la asignatura (información sobre trabajos prácticos). Por otro lado, los alumnos expresaron que el sitio contaba con material suficiente y lo único que había faltado era su propia participación. Además, señalaron la utilidad de los materiales propuestos, aunque expresaron que los utilizaron solo ante determinadas dificultades personales.

\section{Hacia una evaluación general de la propuesta}

A fin de analizar esta experiencia, tomaremos como ejes algunas de las dimensiones propuestas en las encuestas remitidas a los estudiante, a saber, materiales empleados, participación y apreciación general. Asimismo, intentaremos vincular los objetivos de esta estrategia con los resultados obtenidos.

En principio, el espacio en el $F B$ funcionó como un organizador general de la cátedra que permitía tanto la difusión de información como la interacción entre docente y alumnos, la referencia a materiales específicos, el intercambio de diferentes recursos y la discusión a partir de consignas. De este modo, el grupo acompañó la cursada y habilitó un ámbito diferente de comunicación.

El empleo de entradas con consignas que propiciaban la discusión sobre casos de reformulación constituyó tal vez la experiencia más relevante para los objetivos de este trabajo, comoquiera que permitió poner en juego una estrategia didáctica pensada para el fortalecimiento de prácticas de escritura en un ámbito no suficientemente explorado con este fin.

Cabe indicar que los ejemplos empleados resultan especialmente pertinentes dado que recuperan fragmentos textuales de materiales trabajados en las clases, por lo cual los estudiantes podían volver a ellos para recontextualizar los casos sometidos a reformulación y discusión. Asimismo, en el devenir de la comunicación en el $F B$, estas intervenciones particulares del docente proponían la revisión de ejemplos con miras a los primeros parciales que los estudiantes debían enfrentar y habilitaron, incluso, la oportunidad de consultar por mensajería interna al docente, tal como ocurrió en uno de los casos.

$\mathrm{Al}$ respecto, notamos que si bien la participación fue escasa, en las intervenciones observadas se reconoció un potencial significativo que alienta a considerar $y$ fortalecer este recurso.

Sin dudas, el fortalecimiento debe involucrar un trabajo que permita acompañar las intervenciones iniciales de los estudiantes y analizar con ellos representaciones y prácticas vinculadas al $F B$, para constituir a esta red como un recurso al servicio de procesos de enseñanza y aprendizaje. Asimismo, entendemos que un trabajo fuertemente sostenido y articulado entre la clase presencial y la interacción en la red, brindaría a los estudiantes mayor seguridad para intervenir en las propuestas y mejores oportunidades para la revisión de ejes problemáticos del espacio.

\section{Repaso para los parciales en Moodle}

\section{Diseño de la propuesta}

Para la segunda experiencia, se diseńaron repasos para los parciales del TLE. De esta manera, se intentaba dar respuesta a un problema frecuente entre los estudiantes concerniente a las dificultades para estudiar para los parciales como los del TLE, que no evalúan contenidos teóricos sino procedimientos. A partir de este objetivo concreto, se integraron adecuadamente la virtualidad y la presencialidad.

Por otra parte, se utilizó Moodle para el diseño de los materiales y los EVA, ya que esta plataforma era proporcionada por la universidad. Esto daba más institucionalidad a la propuesta y se podía recurrir al personal técnico ante cualquier eventualidad.

Así, en esta plataforma, se armaron dos espacios (uno por cada parcial) en los cuales se incluyeron materiales para repasar los temas de los exámenes. Estos materiales comprendían: 1. documentos (PDF o Power Point) sobre determinados conceptos claves (definición, reformulación, conectores) que los estudiantes debían poner en práctica en los parciales, y 2. cuestionarios de opción múltiple (multiple choice) en los cuales se proponían ejercicios y posibles respuestas. Una vez que los estudiantes resolvían 
los cuestionarios, el sistema les indicaba si su respuesta era correcta o no y por qué, según el caso. También les ofrecía claves de autocorrección; es decir, explicaciones sobre por qué una respuesta era adecuada o no.

El primer espacio, denominado "repaso para el primer parcial” (R1), abarcó cuatro bloques: 1. introducción al espacio y sus características generales; 2 . bloque sobre comprensión de textos explicativos, en el cual figuraban dos textos, con dos cuestionarios; 3. bloque dedicado a la definición, con un documento explicativo sobre el tema y dos cuestionarios en los cuales se ejercitaba la definición, y 4 . bloque dedicado a la reformulación, en el cual se presentó un documento explicativo sobre el tema y diferentes cuestionarios.

El segundo espacio, denominado "repaso para el segundo parcial” (R2), abarcó tres bloques. El primero presentaba una introducción al espacio y los dos restantes se dedicaban al trabajo con diferentes textos. De esta manera, cada bloque incluyó: a. un texto argumentativo; b. un cuestionario dedicado a la comprensión de las ideas clave del texto; c. un cuestionario dedicado a la identificación de los elementos básicos del texto argumentativo (hipótesis y argumentos); $\mathrm{d}$. un cuestionario relativo a errores en el resumen del texto argumentativo, y e. un resumen adecuado del texto leído.

\section{Implementación de la propuesta}

Los repasos se implementaron en cuatro comisiones de la materia, con ciento veinte estudiantes. En una de estas comisiones, los espacios en Moodle se combinaron con un espacio en Facebook, similar al descrito en la experiencia previa.

En relación con el R1, setenta estudiantes ingresaron y cincuenta efectuaron los repasos. En el R2, treinta y ocho estudiantes ingresaron y treinta hicieron los repasos. La mayor cantidad de los estudiantes, en ambos casos, provenían de las comisiones en las cuales se había señalado la obligatoriedad de participar.

Varios estudiantes comunicaron dificultades para ingresar a R1. Esto llevó a que se ofreciera asesoramiento por correo electrónico o presencial para R2.

\section{Discusión sobre ejercicios de reformulación}

Como adelantamos al describir el diseño del R1, hubo una sección dedicada a la reformulación. En esta sección del espacio, se establecieron las siguientes actividades: 1. leer el material titulado La reformulación; 2. leer el material titulado La sinonimia y completar el cuestionario 5 sobre el uso de sinónimos en las reformulaciones; 3 . leer el material titulado Los conectores y completar el cuestionario 6 sobre el uso de conectores en las reformulaciones, y 4 . volver a leer el material La reformulación y completar el cuestionario 7 sobre detección de errores en las reformulaciones.

Los materiales indicados eran documentos, en formato PDF, en los cuales se ofrecían explicaciones sobre los siguientes conceptos: reformulación, sinonimia y conectores, respectivamente. Como hemos mencionado previamente, estos conceptos se ponían en práctica en los parciales y los materiales sistematizaban explicaciones que sobre dichos conceptos se habían ofrecido en clase.

Para los fines de este capítulo y a modo de ejemplo, analizaremos las dificultades que los estudiantes enfrentaron en el cuestionario 7 , en el cual se propuso un fragmento original y una reformulación con errores de dicho fragmento. Posteriormente, se indicaba una serie de alternativas de errores y los estudiantes tenían que seleccionar la alternativa que describiera mejor el error de la reformulación. En ambas consignas, los errores consistieron en la alteración de la relación entre las ideas expresadas en las diferentes cláusulas.

Los resultados mostraron que la principal dificultad de los estudiantes radicaba en el uso de las estrategias léxicas, particularmente el reconocimiento del significado de términos como "acuñar" que aún cuando no sean técnicos no son de uso frecuente en el habla coloquial.

\section{Evaluación del curso por de los estudiantes}

Al finalizar la cursada del TLE, se encuestaron cincuenta y ocho estudiantes de cuatro de las comisiones en las cuales se habían empleado los repasos para los parciales. Estas encuestas apuntaron a reconocer si los estudiantes habían utilizado o no los repasos. Si no los habían utilizado, se indagaba la causa; si los habían utilizado, se les preguntaba acerca de los recursos y herramientas utilizados. En relación con las encuestas, es importante tener en cuenta que en cuanto al porcentaje de participación, tanto en R1 como en R2, participaron más de la mitad de los estudiantes.

En relación con los motivos de la falta de participación, en el caso de R1, el 33,3 \% informó falta de tiempo y otro $33,3 \%$, problemas para el ingreso a la plataforma. En el caso de R2, más del 40 \% comunicó falta de tiempo.

Estos porcentajes nos llevaron a indagar las causas de los problemas para ingresar a la plataforma y concluimos que

$88<$ Universidad de San Buenaventura, Cali - Colombia 
no se trataba de problemas técnicos, sino de dificultades para usar la tecnología. Por este motivo, en experiencias posteriores se planificó el diseño de un instructivo para el ingreso.

Por otra parte, todos los estudiantes que hicieron el repaso destacaron la utilidad de Moodle. Respecto del R1, casi la totalidad indicó que leía la introducción al espacio virtual en la que se indicaba la utilidad del sitio y el modo de utilizarlo. En el caso de R2, la mayoría de los estudiantes (72\%) declararon haber leído la introducción. Un porcentaje menor (16\%) declaró que ya había aprendido el uso del sitio al hacer el primer parcial.

En cuanto a los materiales PDF que incluían ambos repasos, en R1, la mitad de los estudiantes bajaron e imprimieron los materiales para leerlos y la otra mitad leyó de la pantalla. En R2, el 41 \% bajó los materiales y el 59 \% leyó de la pantalla.

En cuanto al uso de los cuestionarios multiple choice, casi todos los estudiantes, en ambos parciales, declararon que para entender mejor el texto leían las devoluciones que recibían sobre el modo como habían resuelto las consignas.

Respecto de la pregunta “¿Agregarías algo al repaso?”, pocos estudiantes propusieron sugerencias.

Para el R1, un estudiante sugirió agregar ejercicios; otro, que se añadan ejercicios diferentes al libro del TLE, con el cual se trabaja en clase, y otro que se añadan textos con otros temas, diferentes a los de ese libro.

Para el R2, tres estudiantes destacaron que les había servido que estuvieran los resúmenes de los textos leídos. Uno de ellos agregó que para reforzar lo hecho había elaborado los resúmenes de los textos.

\section{Hacia una evaluación general de la propuesta}

Para la evaluación de esta experiencia, queremos resaltar muy especialmente el hecho de que fue pensada para dar respuesta a una situación problemática particular, recuperando de este modo las potencialidades de las TIC para brindar oportunidades de aprendizaje por fuera del tiempo presencial del taller. De este modo, la propuesta brinda la posibilidad de diversificar y multiplicar los ámbitos de enseñanza y aprendizaje en torno a las prácticas de lectura y escritura que demandan los estudios superiores y se presenta como una alternativa para andamiar los procesos vinculados a la palabra escrita.
De este modo y dado que las actividades propuestas involucraron tanto recorridos conceptuales como procedimentales, el foco fue puesto en estrategias de lectura y escritura relevantes.

En este punto y frente a la experiencia descrita, la oposición entre obligatoriedad y libertad para la participación de los estudiantes aparece todavía como un aspecto por resolver, que entendemos debe ser fortalecido a partir de una vinculación sostenida y profunda con las actividades del taller, lo cual permitiría ver las interacciones propuestas como una extensión de las prácticas áulicas en la búsqueda de mejores aprendizajes. En esta articulación, el tiempo aparece como un factor condicionante de la participación que entendemos puede estar ligado también a la falta de competencias para la intervención en el EVA propuesto, lo que demandaría un andamiaje sostenido en este aspecto para que los estudiantes puedan hacer un uso óptimo del espacio y de sus potencialidades.

En este punto, aparece como indispensable la necesidad de considerar los conocimientos previos vinculados a las TIC de nuestros estudiantes para planificar estrategias de acompañamiento que fortalezcan la interacción con los recursos incluidos en el EVA.

Por otra parte, entendemos que hubiera sido positivo incluir espacios de foros que permitieran integrar el trabajo individual, de autocorrección, con un trabajo grupal y colaborativo. Sin duda, desde estas dimensiones podemos prever un impacto positivo en el desarrollo de competencias en lectura y escritura vinculadas a los procesos que el taller propicia.

\section{Conclusiones y proyecciones}

Este artículo analizó dos experiencias con el fin de contribuir al desarrollo de las habilidades de reformulación en los estudiantes preuniversitarios a partir de materiales didácticos especialmente diseńados y ofrecidos por las redes de aprendizaje. Consideramos que aun cuando las experiencias son preliminares, su análisis brinda herramientas para repensar el papel de las TIC en propuestas de enseñanza-aprendizaje que apuntan a resolver problemáticas propias de la alfabetización académica.

En principio y en cuanto a la reformulación en sí, cabe destacar que se trata de un procedimiento complejo que involucra habilidades relativas tanto a la lectura como a la escritura. En este sentido, la práctica de dicho procedimiento debe considerar ejercicios que apunten a la lectura y a la comprensión textual, así como a la reformulación 
de algunos fragmentos o de textos completos. El diseño de actividades en los EVA debe tener en cuenta esta observación a fin de organizar los ejercicios en un orden y una disposición que contribuyan a facilitar la resolución de las reformulaciones.

En cuanto al uso de TIC, el $F B$ parecería ser un espacio interesante para encontrarse con los estudiantes. De hecho, la experiencia demuestra que casi la totalidad tiene una cuenta allí y se muestra dispuesta a sumarse a una propuesta que involucre interacciones en la red. Sin embargo, para que este espacio contribuya al mejoramiento de las habilidades de lectura y escritura, debe exponer ejercicios con frecuencia.

También ha mostrado ser productiva la combinación del espacio $F B$ con otro tipo de plataformas como Mood$l e$, que ofrece herramientas para el armado de ejercicios interactivos.

En este contexto, consideramos necesario perfeccionar las estrategias para promover la participación de los estudiantes y generar interacción. Como muestran las experiencias hasta el momento desarrolladas, esta interacción permitiría mejorar las habilidades de reformulación. Cabe destacar en relación con esto, que los propios estudiantes aspiran a ejecutar tareas mediante la interacción grupal como si buscaran la oportunidad de construir un sentido compartido para sus prácticas (Dillon, 2004).

Paralelamente, resultaría fundamental el desarrollo de herramientas multimediales e interactivas para la práctica de estrategias involucradas en la reformulación en particular y las estrategias de lectura y escritura en general. Estas herramientas deben dirigirse al entrenamiento no solo grupal sino también individual (de autocorrección). En este sentido, es interesante el diseño que se propone en la UNGS aún de manera experimental, de ejercicios interactivos para el entrenamiento de las habilidades de comprensión y producción de textos explicativos y argumentativos. Creemos, sin embargo, que se debe continuar en la profundización de una línea de diseño de materiales que aprovechen las potencialidades de la pantalla, a saber, hipertextualidad, multimedialidad e interactividad.

Además de lo mencionado, a la luz del análisis de las dos experiencias resulta indudable la necesidad de integrar adecuadamente la presencialidad y la virtualidad para que los estudiantes no solo ingresen a los sitios y las plataformas, sino que también consulten los materiales propuestos, participen de las discusiones y hagan los ejercicios propuestos. Al hablar de integración, nos referimos a planificar el diseño y el armado de los sitios y materiales virtuales en virtud de los objetivos y problemas didácticos que afronta la materia en cuestión.

Finalmente, proponemos hacer de este artículo una invitación a la reflexión sobre la práctica, para a partir de la revisión de experiencias puntuales, promover el análisis sobre el modo como las TIC pueden contribuir al desarrollo de competencias comunicativas adecuadas a las interacciones con la palabra escrita y hablada que los estudios superiores demandan.

\section{Referencias}

Álvarez, G., García, M. \& Qués, Ma.E. (2010). Entornos virtuales de aprendizaje y didáctica de la Lengua. Una propuesta para mejorar las habilidades de reformulación productiva de estudiantes preuniversitarios. Revista $Q, \mathrm{~N}^{\circ}$ 9, Volumen 5,1-24.

Álvarez, G. \& Cadavid Alvarez, G. (2012). Hacia una propuesta de análisis semiótico integral de ambientes virtuales de aprendizaje. Onomazein, 25 (2012/1), 219-239.

Avgerinou, M. D. (2009). Re-viewing visual literacy in the "Bain d'Images" era- TechTrends, 53 (2), 28-34.

Baldry, A. \& Thibault, P. (2006). Multimodal Transcription and Text Analysis. Londres: Equinox.

Bonaiuti, G. (eds). (2006). E-Learning 2.0. Il futuro dell'apprendimento in rete, tra formale e informale. Lavis: Edizione Erickson.

Carlino, P. (2005). Escribir, leer y aprender en la universidad. Una introducción a la alfabetización académica. Buenos Aires: Fondo de Cultura Económica

Dillon, P. (2004). Trajectories and tensions in the theory of information and communication technology in education. British Journal of Educational Studies Vol. 52 (2), 138-150.

Estienne V. \& Carlino P. (2004). Leer en la universidad. Enseñar y aprender una cultura nueva. $7^{\circ}$ Congreso Internacional de promoción de la Lectura y el Libro. Buenos Aires.

García, M. \& Álvarez, G. (2009). La reformulación de texto fuente en alumnos de nivel preuniversitario: una propuesta superadora de las dificultades en la producción del texto escrito. Actas de las Jornadas de Enseñanza de la Lengua y la Literatura. Leer y escribir: nuevas miradas sobre viejas prácticas. Los Polvorines: Universidad Nacional de General Sarmiento.

García, M. \& Álvarez, G. (2010). Hacia una propuesta superadora de las dificultades de alumnos preuniversitarios en reformulaciones productivas del texto fuente. Onomazein 21 (1): 191-223.

Granieri, G. (2005). Blog generation. Bari: Editori Laterza.

Gros Salvat, B. (2000). El ordenador invisible. Hacia la apropiación del ordenador en la enseñanza. Barcelona: Editorial Gedisa

$90<$ Universidad de San Buenaventura, Cali - Colombia 
Gros Salvat, B. (2004). La construcción del conocimiento en la red: límites y posibilidades. Teoría de la educación: educación y cultura en la sociedad de la información No 5.

Harasim, L.; Hiltz R.; Turoff, M. \& Teles, L. (1998). Redes de aprendizaje. Guía para la enseñanza y el aprendizaje en red. Barcelona: Editorial Gedisa.

Kress, G. (2004). Reading Images: Multimodality, Representation and New Media. Disponible en http://www.knowledgepresentation.org/BuildingTheFuture/Kress2/Kress2.html. [Consultado el 10 de marzo de 2015]

Kress, G. (2010). Multimodality. A social semiotic approach to contemporary Communications. Londres: Routledge.

Lacon de De Lucia, N. \& Ortega de Hocevar, S. (2004). La problemática de la escritura en la universidad: una propuesta de solución a partir de la articulación con el Polimodal. I Congreso Internacional Educación, Lenguaje y Sociedad: "Tensiones Educativas en América Latina”. Santa Rosa (Argentina): Facultad de Ciencias Humanas, Universidad Nacional de La Pampa.

Laurillard, D. (2010). Effective Use of Technology in Teaching and Learning in HE. International Encyclopedia of Education, 4, 419-426.

Llorens, F; Capdeferro, N. (2011). Posibilidades de la plataforma Facebook para el aprendizaje colaborativo en línea. Revista de Universidady Sociedad del Conocimiento (RUSC). Vol. 8, n.ş 2, págs. 31-45. UOC.

Martínez, M. C. (2002). Estrategias de lecturas y escritura de textos. Perspectivas teóricas y talleres. Cátedra UNESCO Lectura y Escritura. Editorial Taller de Artes gráficas Facultad de Humanidades, Universidad del Valle.

Martínez, M. C. (2006). Curso virtual de comprensión y composición de textos escritos. Cátedra Unesco Lectura y Escritura, Dintev y Gobernación del Valle. Universidad del Valle. Cali, Colombia.

Miles, M. B.; Huberman, AM. M. (1991). Analyse des données qualitatives. Receuil de nouvelles méthodes. París: De Boeck Université.

Narvaja de Arnoux, Di Stefano, E \& M., Pereira, C. (2002). La lectura y la escritura en la universidad. Buenos aires: EUDEBA.

Neiman, G.; Quaranta, G. (2006). Los estudios de caso en la investigación sociológica. En: Vasilachis de Gialdino, I. (coord.) Estrategias de investigación cualitativa (213-238). Barcelona: Gedisa.

Parodi, G. (2003). Relaciones entre lectura y escritura: una perspectiva cognitiva discursiva. Antecedentes teóricos y resultados empiricos. Valparaíso: Ediciones Universitarias de Valparaíso.

Parodi, G. (2005). Comprensión de textos escritos, Buenos Aires: Eudeba.

Piacente, T. \& Tittarelli, A. M. (2006). Comprensión y producción de textos en alumnos universitarios: la reformulación textual. Orientación y Sociedad Vol. 6, 99-126.
Reale, A. (2008). "Sabía que me gustaba escribir, pero nunca pensé que fuera para tanto". Sobre la eficacia de bitácoras y diarios de escritor en el Taller de Expresión I. Jornadas Académicas 2008 "Producir teoría, pensar las prácticas". Buenos Aires: Universidad de Buenos Aires.

Rey Valzacchi, J. (2010). Educación virtual en Argentina. En Torres, P. \& Rama, C. 2010. La educación superior a distancia en América Latina y el Caribe. Santa Catarina: Editora Unisul.

Rodríguez Illera, J.L. \& Escofet Roig, A. (2006). Clasificaciones del aprendizaje híbrido y criterios de buenas prácticas universitarias. Universidad Nacional, Costa Rica. Aprendizaje Virtual y Desarrollo Sostenible: El Rol de las Universidades (pp. 1-65, 67). San José, Costa Rica: Universidad Nacional, Costa Rica.

Salaverría, R. (2001). Aproximación al concepto de multimedia. Desde los planos comunicativo e instrumental. Estudios sobre el mensaje periodistico N. ${ }^{\circ}$ 7, 383-395.

Scardamalia, M. \& Bereiter, C. (1992). Dos modelos explicativos de los procesos de composición escrita. Infancia y aprendizaje, 58, 43-64.

Scolari, C. 2004. Hacer clic. Barcelona: Gedisa.

Scolari, C. (2008). Hipermediaciones: elementos para una teoría de la comunicación digital interactiva. Buenos Aires: Gedisa.

Scolari, C. (2009). Desfasados. Las formas de conocimiento que estamos perdiendo, recuperando y ganando. Versión No $22,163-185$

Silvestri, A. (1998). En otras palabras. Las habilidades de reformulación en la producción del texto escrito. Buenos Aires: Cántaro.

Taboada, M. (2011). Escribir en el nivel superior: pretextos para un compromiso. Prisma Revista de Didáctica, No1, 35-40.

Taboada, M., Breganni, R., Cejas, M. \& Spada, M. (2010). Leer y escribir en la Universidad: desafíos y reflexiones para revisar las prácticas. Actas de las VII Jornadas Pedagógicas del Bicentenario. Concepción del Uruguay (Argentina): Facultad de Humanidades, Artes y Ciencias Sociales, Universidad Autónoma de Entre Ríos.

Torres, P. \& Rama, C. (2010). La educación superior a distancia en América Latina y el Caribe. Santa Catarina: Editora Unisul.

Van Dijk, T. \& Kintsch, W. (1983). Strategies of discourse comprehension. New York: Academic Press.

Vasilachis de Gialdino, I. (coord.) (2006). Estrategias de una investigación cualitativa. Buenos Aires: Editorial Gedisa.

Vázquez, A. \& Miras, M. (2004). Cómo se representan estudiantes universitarios las tareas de escritura. Reunión Mente y Cultura: Cambios Representacionales en el Aprendizaje. Centro Regional Universitario Bariloche de la Universidad Nacional del Comahue. Bariloche. 